\title{
CsGSTU8, a Glutathione S-Transferase From Camellia sinensis, Is Regulated by CsWRKY48 and Plays a Positive Role in Drought Tolerance
}

\author{
Yongheng Zhang, Jingyuan He, Yezi Xiao, Yingao Zhang, Yingqin Liu, Siqing Wan, \\ Lu Liu, Yuan Dong, Huan Liu and Youben Yu* \\ College of Horticulture, Northwest A\&F University, Xianyang, China
}

\section{OPEN ACCESS}

Edited by:

Rajeev K. Varshney, International Crops Research Institute for the Semi-Arid Tropics (ICRISAT),

India

Reviewed by:

Xinchao Wang,

Tea Research Institute, Chinese Academy of Agricultural Sciences

(CAAS), China

Huiying Li,

Wuhan Botanical Garden, Innovative Academy of Seed Design (INASEED), Chinese Academy of Sciences (CAS),

China

*Correspondence: Youben Yu yyben@163.com

Specialty section: This article was submitted to

Plant Abiotic Stress,

a section of the journal

Frontiers in Plant Science

Received: 15 October 2021 Accepted: 17 November 2021 Published: 09 December 2021

Citation:

Zhang Y, He J, Xiao Y, Zhang Y, Liu Y, Wan S, Liu L, Dong Y, Liu H and Yu Y (2021) CsGSTU8, a Glutathione S-Transferase From Camellia sinensis, Is Regulated by CsWRKY48 and Plays a Positive Role in Drought Tolerance.

Front. Plant Sci. 12:795919. doi: 10.3389/fpls.2021.795919
Glutathione S-transferases (GSTs) constitute a large family of enzymes with a wide range of cellular functions. Recently, plant GSTs have gained a great deal of attention due to their involvement in the detoxification of electrophilic xenobiotics and peroxides under adverse environmental conditions, such as salt, cold, UV-B and drought stress. A previous study reported that a GST gene (CSGSTU8) in tea plant was distinctly induced in response to drought, suggesting this gene plays a critical role in the drought stress response. In this study, by using quantitative real-time PCR (qRT-PCR) and $\beta$-glucuronidase (GUS) reporter lines, we further demonstrated that CSGSTU8 was upregulated in response to drought stress and exogenous abscisic acid (ABA) treatments. Overexpression of CSGSTU8 in Arabidopsis resulted in enhanced drought tolerance as indicated by the improved scavenging of excess amounts of reactive oxygen species (ROS) under drought conditions. Furthermore, we found that CsWRKY48 acts as a transcriptional activator and that its expression is induced in response to drought stress and ABA treatment. Electrophoretic mobility shift assays (EMSAs), dual-luciferase (LUC) assays and transient expression assays in tea plant leaves revealed that CsWRKY48 directly binds to the W-box elements in the promoter of CSGSTU8 and activates its expression. Taken together, our results provide additional knowledge of drought stress responses in tea plant.

Keywords: Camellia sinensis, glutathione S-transferases (GSTs), ROS, WRKY TF, drought stress

\section{INTRODUCTION}

Adverse environmental conditions, especially drought conditions, greatly limit plant growth and development (Shao et al., 2008). Plants have evolved intricate defensive systems to survive under drought-stress conditions, specifically, regulating the balance of reactive oxygen species (ROS) (including hydrogen peroxide $\left(\mathrm{H}_{2} \mathrm{O}_{2}\right)$, superoxide $\left(\mathrm{O}^{2-}\right), \mathrm{OH}^{\bullet}$ and $\left.{ }^{1} \mathrm{O}_{2}\right)$ in cells is an indispensable strategy (Carvalho, 2008; Singh et al., 2019). On the one hand, ROS are necessary for plant growth, as plants actively produce ROS that serve as signal transduction molecules for growth under suitable conditions. On the other hand, when plant experience drought stress, excessive amounts of ROS 
accumulate in cells and cause irreversible damage to membranes, proteins, and RNA and DNA molecules, even resulting in oxidative destruction of the cells (Moller et al., 2007; Choudhury et al., 2017). Thus, accelerating the expression or activities of antioxidant enzymes and antioxidants, which are involved in ROS scavenging, is critical for the drought tolerance of plants (Smirnoff, 1993; Gill and Tuteja, 2010; Qi et al., 2018).

Glutathione S-transferases (GSTs) constitute a large family of enzymes with a wide range of cellular functions in plants, including protecting organisms against oxidative stress under stress conditions by participating in ROS scavenging (Jha et al., 2011; Rong et al., 2014; Qi et al., 2018). Plant GSTs can be divided into eight distinct subclasses according to their protein sequence and function: Phi (GSTF), Tau (GSTU), Theta (GSTT), Zeta (GSTZ), Lambda (GSTL), Elongation factor 1 gamma (EF1G), DHAR, and TCHQD proteins (Sasan et al., 2011). Recently, several classes of GSTs, including Tau GSTs, have received a great deal of attention in plants due to the assistance of these GSTs in regulating the metabolism of oxidized molecules under drought conditions (Liu et al., 2013b; Xu et al., 2016; Choudhury et al., 2017). The expression level of GSTs in plants was reported to be positively correlated with the rate of oxidized molecule scavenging and to contribute to drought stress tolerance in previous studies (Kumar et al., 2013; Stavridou et al., 2021). For example, transgenic Arabidopsis plants overexpressing tomato LeGSTU2 show enhanced resistance to drought stress via increased antioxidative enzyme activities for scavenging excess ROS (Xu et al., 2015). Liu et al. (2013a) reported that overexpression of a zeta GST gene from Pyrus pyrifolia in tobacco improved $\mathrm{O}^{2-}$ scavenging under drought conditions, thus resulting in increased tolerance to drought. Moreover, overexpression of a GST gene (ThGSTZ1) from Tamarix hispida improves drought tolerance by enhancing the ability to scavenge ROS (Yang et al., 2014).

Although GSTs have been observed to protect cells from oxidative stress under drought-stress conditions, knowledge about the intricate regulation of GSTs under drought stress remains scarce. To date, several GSTs have been reported to be directly activated by specific transcription factors to regulate drought stress tolerance. For example, in wheat, the BES/BZR family transcription factor TaBZR2 functions positively in the drought response by activating TaGST1 to scavenge droughtinduced $\mathrm{O}_{2}{ }^{-}$accumulation (Cui et al., 2019). A wheat ethyleneresponse factor, TaERF3, was also reported to directly activate the expression of TaGST6 by binding to the TaGST6 promoter to improve drought tolerance (Rong et al., 2014). Furthermore, Ren et al. (2020) demonstrated that a NAC transcription factor, ZmNST3, enhances maize drought stress tolerance by directly binding to the promoters of GST and GlnRS and activating their expression.

Camellia sinensis is a commercially important perennial evergreen woody crop species that is widely cultivated worldwide and is highly susceptible to drought stress (Parmar et al., 2019). Although several tea plant GSTs have been reported to be involved in cold and drought stress responses based on their expression levels (Parmar et al., 2019; Samarina et al., 2020; Sun et al., 2020), the roles of tea plant GSTs in drought stress are still poorly understood. CsGSTU8 was found to be strongly induced in response to drought stress in previous studies (Xia et al., 2019) and according to our transcriptome data (unpublished), thus prompting us to investigate its role in drought stress. In this study, we used quantitative real-time PCR (qRT-PCR) and transgenic $\beta$-glucuronidase (GUS) reporter lines to confirm the upregulation of CsGSTU8 under drought and abscisic acid (ABA) treatments. Furthermore, transgenic Arabidopsis plants expressing CsGSTU8 showed improved ROS scavenging, thus contributing to drought resistance. In addition, CsWRKY48, a tea plant transcriptional activator, whose expression is induced in response to drought and ABA treatment, can directly bind to the promoter and activate the expression of CsGSTU8. Taken together, our findings provide evidence for the positive role of the GSTU8 protein in drought stress and are helpful for understanding the mechanism of the drought response in tea plant.

\section{MATERIALS AND METHODS}

\section{Plant Materials and Treatment}

Hydroponically grown Longjing-changye annual tea seedlings were preincubated in a greenhouse at Northwest A\&F University Yangling $\left(34^{\circ} 20^{\prime} \mathrm{N}, 108^{\circ} 24^{\prime} \mathrm{E}\right)$, Shaanxi Province, China, under natural light, and the temperature was maintained at $20 / 25^{\circ} \mathrm{C}$ in the dark/light. Seedlings displaying consistent growth were divided into three treatment groups: normal, $15 \%$ polyethylene glycol (PEG) 6,000 and ABA $(100 \mu \mathrm{M})$ treatments. The first to third leaves were collected at $0,4,8,12,24$, and $48 \mathrm{~h}$ after treatment for RNA extraction. For all the treatments, three biological replicates were included.

\section{RNA Extraction and qRT-PCR Analysis}

Total RNA extraction and qRT-PCR were carried out as previously described by Wan et al. (2020). The primers used in the expression analysis are listed in Supplementary Table 1.

\section{Transformation of Arabidopsis With CsGSTU8 and Subcellular Localization of CsGSTU8}

The CDS of CsGSTU8 was amplified from the cDNA of Longjingchangye tea plant according to the sequence information reported in the Tea Plant Information Archive (TPIA) (Xia et al., 2019). Then, the Kpn I and BamH I sites of pCAMBIA2300-GFP vector were selected to generate 35S::CsGSTU8-GFP construct. To obtain transgenic Arabidopsis, Agrobacterium tumefaciens strain GV3101 harboring the 35S::CsGSTU8-GFP construct was transformed into wild-type (WT) Arabidopsis ecotype Columbia (Col-0) by using the floral-dip method (Zhang et al., 2006). T3generation homozygous lines (OE3 and OE7) and Col-0 (WT) plants were used for further analysis.

To determine the subcellular localization of the CsGSTU8 protein, GV3101 cells harboring 35S::CsGSTU8-GFP and 35S::GFP were cultured and adjusted to an OD600 of 0.6 via an infiltration buffer $\left(10 \mathrm{mM}\right.$ MES, $10 \mathrm{mM} \mathrm{MgCl}_{2}$ and 100 
$\mu \mathrm{M}$ acetosyringone; $\mathrm{pH}$ 5.7). After $2 \mathrm{~h}$ incubation at $25^{\circ} \mathrm{C}$, the cultures were transiently expressed in 4-week-old tobacco (Nicotiana benthamiana) leaves, and the GFP signal was observed with a BX63 Automatic Fluorescence Microscope (Olympus, Japan) at $48 \mathrm{~h}$ after infiltration. The primers used are listed in Supplementary Table 1.

\section{Stress Tolerance Assays}

Transgenic and WT Arabidopsis seeds were surface sterilized with $20 \% \mathrm{NaClO}$ (including $0.1 \%$ Triton X-100) and sown on Murashige and Skoog (MS) agar media that included mannitol $(0,100,125$, and $150 \mathrm{mM})$. Media containing seeds were placed in a controlled growth chamber under $16 / 8 \mathrm{~h}\left(25 / 20^{\circ} \mathrm{C}\right)$ day/night conditions for growth after incubation at $4{ }^{\circ} \mathrm{C}$ for 3 days. The survival rate was calculated after 6 -days (normal conditions) or 10-days (mannitol conditions) of growth. Seedlings with green leaves under mannitol conditions were considered alive.

For drought treatment in soil, WT and transgenic seeds were germinated in soil (vermiculite: humus $=1: 1$ ) and grown under normal conditions for 7 days. Then watering was withheld until drought stress symptoms occurred. Relative electrolyte leakage was measured to compare the drought tolerance of WT and transgenic seedings following previously report (Jiang et al., 2019).

\section{Measurement of Reactive Oxygen Species Contents, Malondialdehyde Contents and Glutathione S-Transferase Activity}

3,3'-Diaminobenzidine (DAB) staining and nitro blue tetrazolium (NBT) staining were performed to detect the accumulation of $\mathrm{H}_{2} \mathrm{O}_{2}$ and $\mathrm{O}^{2-}$ in the leaves according to our previously described methods (Zhang et al., 2020). The contents of $\mathrm{H}_{2} \mathrm{O}_{2}$ and $\mathrm{O}^{2-}$ were measured following the previous report (Shi et al., 2010). To determine the Malondialdehyde (MDA) content, a thiobarbituric acid (TBA) reaction was performed according to a previously described method (Draper et al., 1992). The GST activity was measured according to the protocol provided with a glutathione-S-transferase Assay Kit (BCO350, Solarbio, China).

\section{$\beta$-Glucuronidase Reporter Construction and $\beta$-Glucuronidase Activity Assays}

The 1,500 bp promoter of CsGSTU8 was inserted into a pCAMBIA1300-GUS vector (Hind III and BamH I) to activate the GUS gene. Then, the construct was transformed into WT Arabidopsis; 7-day-old T2-generation seedings were treated with ABA $(1 \mu \mathrm{M})$ and dehydrated (20\% PEG 6,000). After $12 \mathrm{~h}$ of treatment, GUS staining and activity were measured as described previously (Jefferson et al., 1987). The primers used are listed in Supplementary Table 1.

\section{Electrophoretic Mobility Shift Assays}

Full-length CsWRKY48 (TEA008513) was inserted into a pGEX4T-1 vector to construct a GST-CsWRKY48 expression vector, and the recombinant plasmid was transformed into
E. coli strain Rosetta2 (EC1014, Weidi Biotechnology, Shanghai, China). Expression of the GST and GST-CsWRKY48 proteins was induced by $1 \mathrm{mM}$ isopropyl-b-D-thiogalactoside (IPTG) at $16^{\circ} \mathrm{C}$ for $20 \mathrm{~h}$, and then the fusion proteins were purified using glutathione Sepharose (GSTrap ${ }^{\text {TM }}$ HP, GE Healthcare, United States). A biotin-labeled probe containing "W-box" elements in the promoter of CsGSTU8 was synthesized by Tsingke. EMSAs were carried out according to the protocol provided with a chemiluminescent EMSA kit (GS009, Beyotime Biotechnology, China).

\section{Transient Transcriptional Activation Assays}

The 1,500 bp promoter fragment of CsGSTU8 was inserted into the luciferase (LUC) reporter plasmid pGreen II 0800, which contains a Renilla luciferase (REN) gene under the control of the $35 S$ promoter used as an internal control. The 35S::CsWRKY48GFP vector as effector. The effector plasmids and the reporter plasmids were transformed into GV3101 (pSoup), and the effector and corresponding reporter in GV3101 (pSoup) were mixed together at a proportion of 2:1 and subsequently injected into tobacco leaves. Forty-eight hours later, the LUC signal was visualized with a CCD system (Lumazone Pylon 2048B, Princeton, United States). The activities of LUC and REN were determined according to the protocol of a dual-Luciferase reporter assay kit (FR201, TransGen Biotech, China) by using a full-wavelength multifunctional enzyme labeling instrument (Tecan Infinite M200PRO, Tecan, Switzerland).

Two-month-old Fudingdaba tea plants grown from seeds in a growth chamber were selected for transient expression in accordance with the methods of Shui et al. (2021) with slight modifications. A. tumefaciens strain GV3101 harboring 35S::CsWRKY48-GFP and 35S::GFP was injected into the different sides of the same leaf. At 3 days after injection, leaves were collected for RNA isolation, and RT-PCR and qRT-PCR were then carried out to assess the transformation results and the measure expression of CsGSTU8, respectively. The primers used are listed in Supplementary Table 1.

\section{Statistical Analysis}

SPSS 19.0 software was used for statistical data analysis. Data for tissue-specific expression, survival rate and relative electrolyte leakage were subjected to one-way analysis of variance, and the differences between means were assessed by Duncan's multiple range tests. Other data were examined by Student's $t$-tests. The values are represented as means \pm standard deviations.

\section{RESULTS}

\section{Identification, Expression in Different Tissues and Subcellular Localization of CsGSTU8}

Our transcriptome data (Supplementary Figure 1) and those from the TPIA ${ }^{1}$ revealed that TEA019065 was strongly responsive

\footnotetext{
${ }^{1}$ http://tpia.teaplant.org/
} 


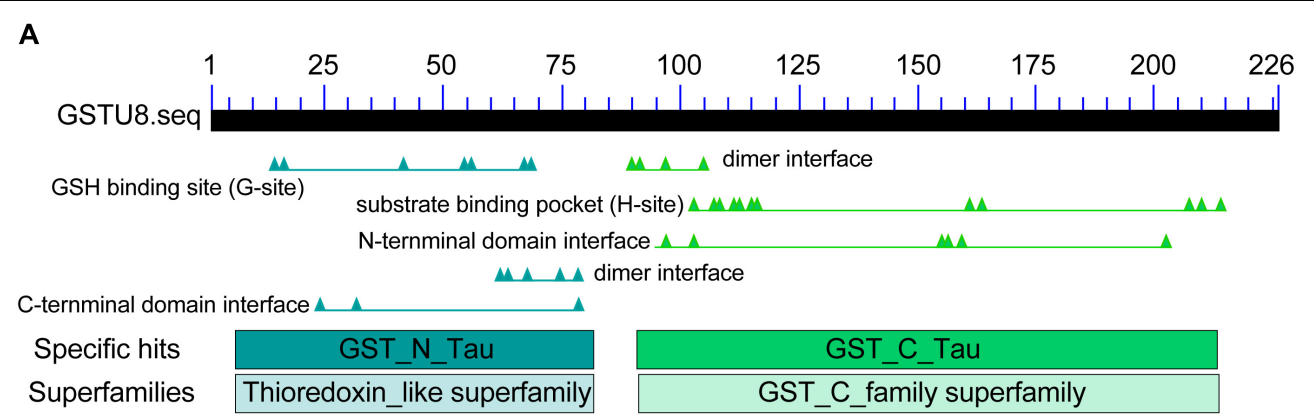

B
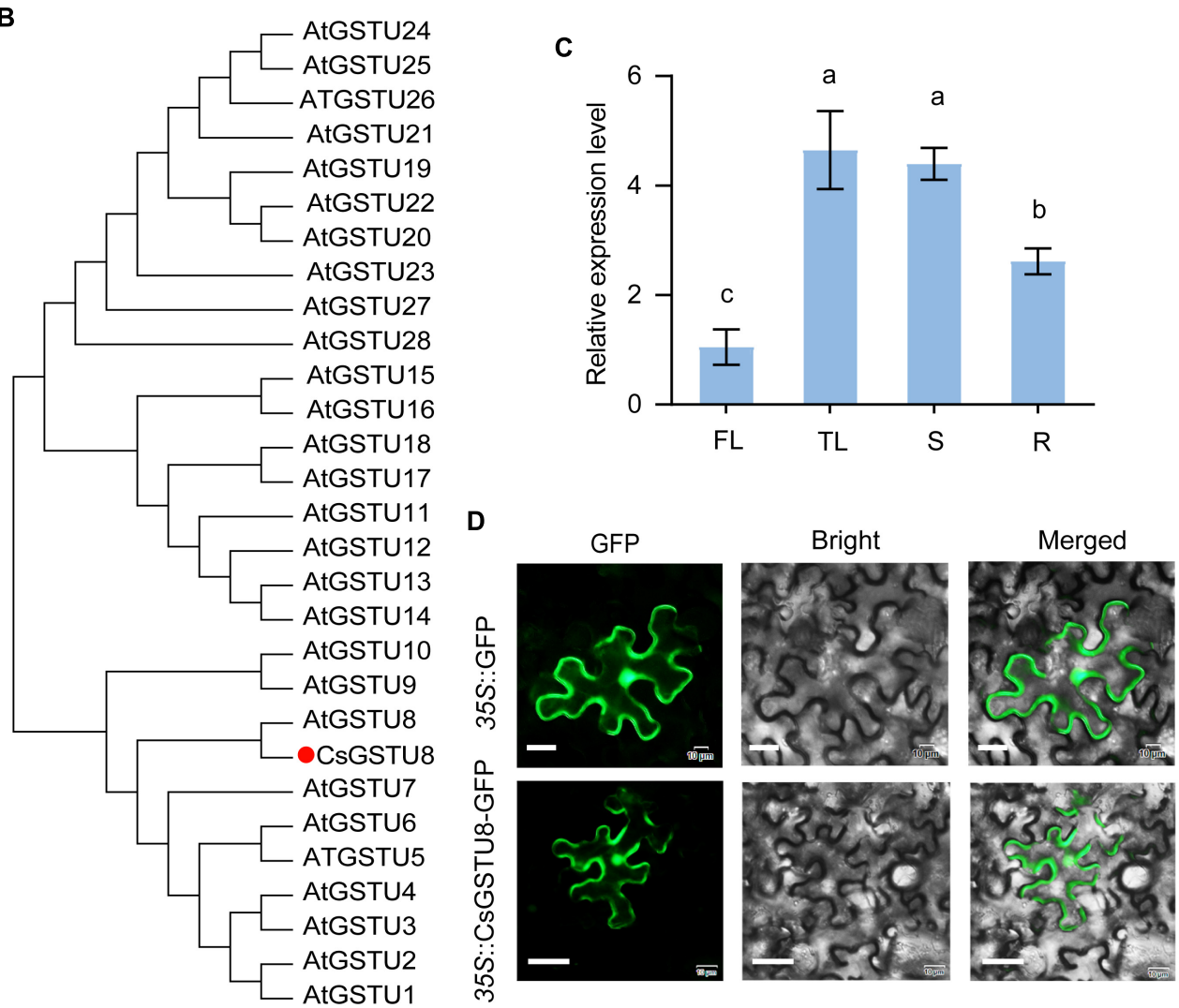

FIGURE 1 | Characterization of CsGSTU8 from Camellia sinensis. (A) Conserved domain analysis of the CsGSTU8 protein sequence retrieved from the NCBI database (https://www.ncbi.nlm.nih.gov/Structure/cdd/). (B) Phylogenetic analysis of CsGSTU8 and GSTU proteins from Arabidopsis. (C) Expression of CsGSTU8 in different tissues. FL (first leaf), TL (third leaf), S (stems), R (root tips). The data are the means \pm SDs of three independent experiments. The values not followed by the same letter are significantly different according to Duncan's multiple range test $(P<0.05)$. (D) Subcellular localization of 35S::CsGSTU8-GFP and 35S::GFP in tobacco cells,. Bar $=20 \mu \mathrm{m}$.

to drought stress (Xia et al., 2019). In addition, it was also reported to be distinctly induced in response to drought stress in both drought-tolerant and drought-sensitive tea plants (Parmar et al., 2019). These findings prompted us to investigate the role of this gene in drought stress. Therefore, we isolated the complete CDS of TEA019065 from Longjing-changye seedings and found that it encodes a GST belonging to the Tau subfamily and harbors a GSH-binding site in the N-terminal region (Figure 1A). The protein comprises 226 amino acids and has a molecular weight of $26.36 \mathrm{kDa}$. Phylogenetic analysis including all Tau GST proteins from Arabidopsis showed that TEA019065 is closely related to AtGSTU8 (Figure 1B); hence, it was named CsGSTU8 in this study.

To explore the expression levels of CsGSTU8 in different tissues, we investigated its expression in the first leaves, third leaves, stems and root tips via qRT-PCR. The results showed that CsGSTU8 tended to expressed greater in the mature organization (the third leaves and stem) than young organs (the first leaves and root tips) (Figure 1C).

A. tumefaciens strain GV3101 harboring 35S::CsGSTU8-GFP and 35S::GFP was introduced into tobacco leaves to investigate the subcellular localization of the CsGSTU8 protein. The 


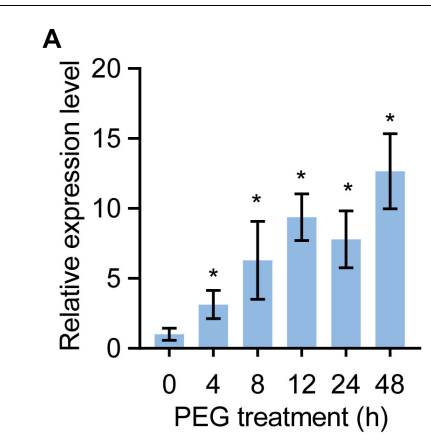

C

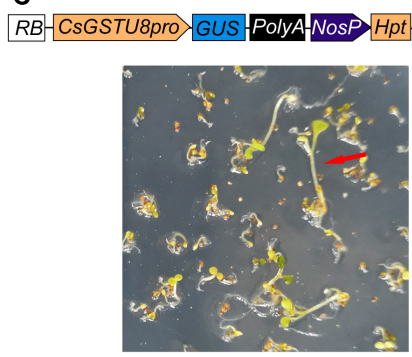

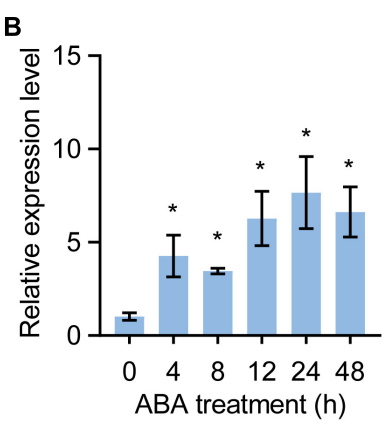

D

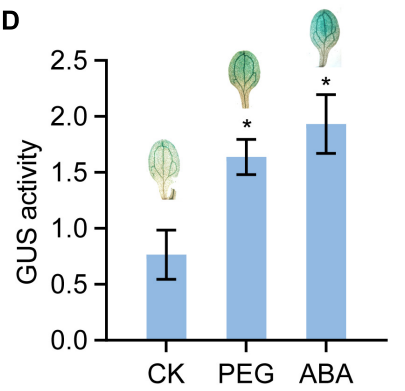

FIGURE 2 | Expression patterns of CSGSTU8 under PEG and ABA treatments. (A) Expression of CSGSTU8 after PEG treatment. (B) Expression of CsGSTU8 under ABA treatment. (C) Construction of CsGSTU8pro::GUS transgenic lines. Hygromycin B was used to screen transgenic Arabidopsis plants. (D) Measurement of the GUS activities in CsGSTU8pro::GUS transgenic Arabidopsis leaves under PEG and ABA treatments. The Data are presented as the means \pm SDs of three independent experiments. Significant differences were determined using Student's $t$-test $\left({ }^{*} P<0.05\right)$.

fluorescence of GFP fused to CsGSTU8 showed no difference from that of the GFP control, which means that the CsGSTU8 protein was distributed throughout the cell (Figure 1D).

\section{Expression Analysis of CsGSTU8 Under Drought and Abscisic Acid Treatments}

To validate the drought response of CsGSTU8, tea plants were treated with $15 \%$ PEG 6,000 to mimic drought stress, and the transcript levels of CsGSTU8 were measured by qRT-PCR. The results showed that the expression of CsGSTU8 gradually increased in response to drought stress (Figure 2A). Notably, ABREs involved in ABA responsiveness were found in the promoter of CsGSTU8 (Supplementary Figure 2), implying that the CsGSTU8 expression is induced in response to ABA treatment. qRT-PCR was used to measure the expression of CsGSTU8 under ABA treatment, and as expected, ABA induced the expression of CsGSTU8 (Figure 2B). To better understand CsGSTU8 expression patterns, a CsGSTU8pro::GUS transgenic line was generated and subjected to drought and ABA; the results showed that GUS activity significantly increased under drought stress and ABA treatment (Figures 2C,D). Taken together, these results further reveal that the expression of CsGSTU8 was induced in response to drought stress and ABA treatment.

\section{CsGSTU8 Confers Drought Tolerance to Transgenic Arabidopsis Plants}

Transgenic Arabidopsis plants were generated to explore the function of CsGSTU8 in tolerance to drought stresses. Two T3-generation homozygous lines (OE3 and OE7) with high expression of CsGSTU8 gene were selected for further analysis (Supplementary Figure 3). Various concentrations of mannitol were employed to mimic drought stress. As expected, in the absence of mannitol condition, the survival rates of the two transgenic lines were indistinguishable from those of the WT (Figures 3A,B). The application of mannitol inhibited the growth of both the WT and transgenic lines in a dose-dependent manner; however, the survival rates of the OE3 and OE7 lines significantly increased compared to those of the WT plants under 100, 125, and $150 \mathrm{mM}$ mannitol conditions (Figures 3A,B).

Moreover, under soil water shortage conditions, the WT plants exhibited a more severely wilted phenotype than did the OE3 and OE7 transgenic lines (Figure 3C). We further measured the relative electrolyte leakage to evaluate membrane damage in Arabidopsis leaves, and the results showed that there was no difference between the WT and transgenic plants under normal conditions, while under drought stress, the relative electrolyte leakage of the two transgenic lines was lower than that of the WT (Figure 3D). These results indicate that overexpression of CsGSTU8 enhances drought tolerance in Arabidopsis.

\section{Overexpression of CsGSTU8 Alleviates Reactive Oxygen Species and Malondialdehyde Accumulation in Arabidopsis}

As it is well established that GSTs contribute to alleviating excess amounts of ROS when plants experience stress (Sharma et al., 2014; Yang et al., 2014), we evaluated the GST activity and 

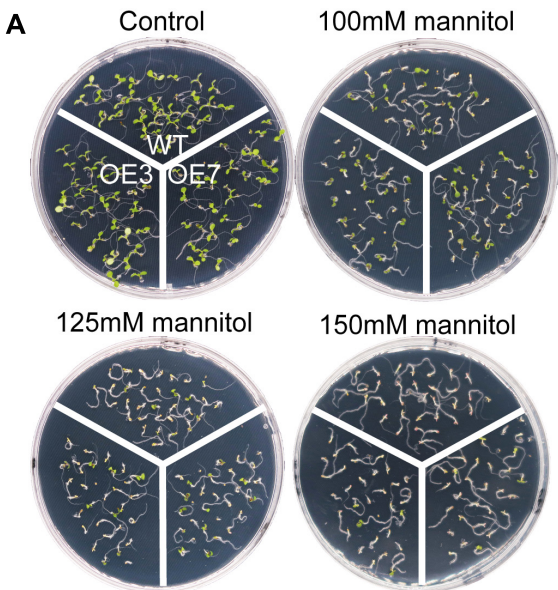

150mM mannitol

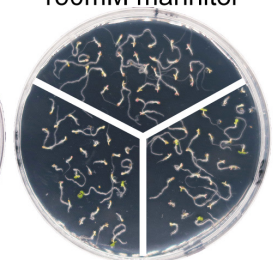

B

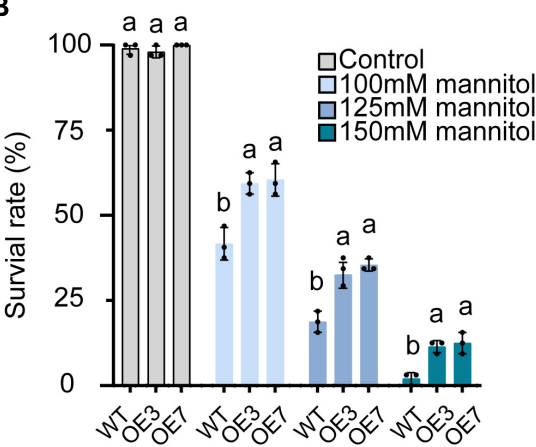

C

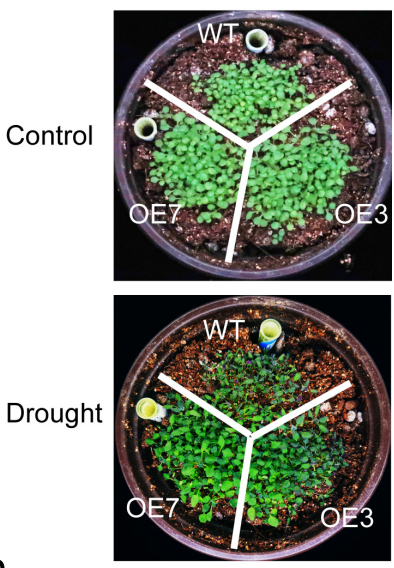

D

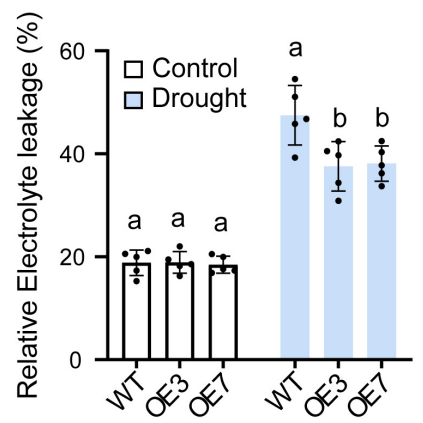

FIGURE 3 | Overexpression of CSGSTU8 improves drought stress tolerance in transgenic Arabidopsis. (A) Growth phenotypes under control and mannitol conditions in MS media. (B) Measurement of survival rate in MS media. The Data are presented as the means \pm SDs of three independent experiments. (C) Growth phenotypes under control and drought stress conditions in the soil. (D) Measurement of relative electrolyte leakage. The Data are the means \pm SDs of five independent experiments. The Values not followed by the same letter are significantly different according to Duncan's multiple range test $(P<0.05)$.

ROS content to explore whether enhanced drought tolerance is associated with a decrease of ROS levels in transgenic lines. The results showed that GST activity was higher in the transgenic plants than in the WT plants under both control and drought conditions (Figure 4B). Interestingly the contents of $\mathrm{H}_{2} \mathrm{O}_{2}$ and $\mathrm{O}^{2-}$ in the WT and transgenic lines were not significantly different under normal conditions, while under drought stress, the OE3 and OE7 transgenic lines accumulated less $\mathrm{H}_{2} \mathrm{O}_{2}$ and $\mathrm{O}^{2-}$ than the WT plants did (Figures $4 \mathrm{~A}, \mathrm{C}, \mathrm{D}$ ). MDA is considered a marker of membrane lipid peroxidation caused by excessive ROS (Jambunathan, 2010), so we also measured the MDA content of WT and transgenic lines under drought stress. The transgenic lines consistently accumulated lower MDA than did the WT under drought conditions (Figure 4E). These results implying that CsGSTU8 overexpression in Arabidopsis alleviates ROS and MDA accumulation under drought stress.

\section{CsWRKY48 Is a Transcriptional Activator Involved in Responses to Drought and Abscisic Acid in Tea Plant}

Our team previously reported that CsWRKYIIc3 (annotated as CsWRKY48 in the TPIA) is involved in responses to drought and ABA in tea plant Shaancha No. 1 tea plant (Xiao et al., 2020).
Our unpublished transcriptome data also demonstrated that CsWRKY48 expression was induced in response to drought (Supplementary Figure 1). In this study, qRT-PCR was used to explore whether CsWRKY48 responds to drought and ABA treatment in Longjing-changye seedings, and the results showed that CsWRKY48 was indeed induced in responses to drought and ABA treatment (Figures 5C,D). Amino acid sequence analysis and subcellular localization results revealed that CsWRKY48 contains a highly conserved WRKY DNA-binding domain (Figure 5A) and localizes to the nucleus (Figure 5E), so we fused the CDS of CsWRKY48 with the Gal4-binding domain in the pGBKT7 vector, after which the construct was transferred to yeast strain $\mathrm{Y} 2 \mathrm{H}$ Gold to investigate its transactivation capacity. The results showed that the fusion of CsWRKY48 with Gal4 was able to activate HIS3, ADE2 and MEL1 and grow on selective media (Figure 5B), indicating that CsWRKY48 has potential transcriptional activation.

\section{CsWRKY48 Bind to the CsGSTU8 Promoter and Activates the Expression of CsGSTU8}

W-boxes were found in the promoter of CsGSTU8 (Supplementary Figure 2), implying that the expression of 

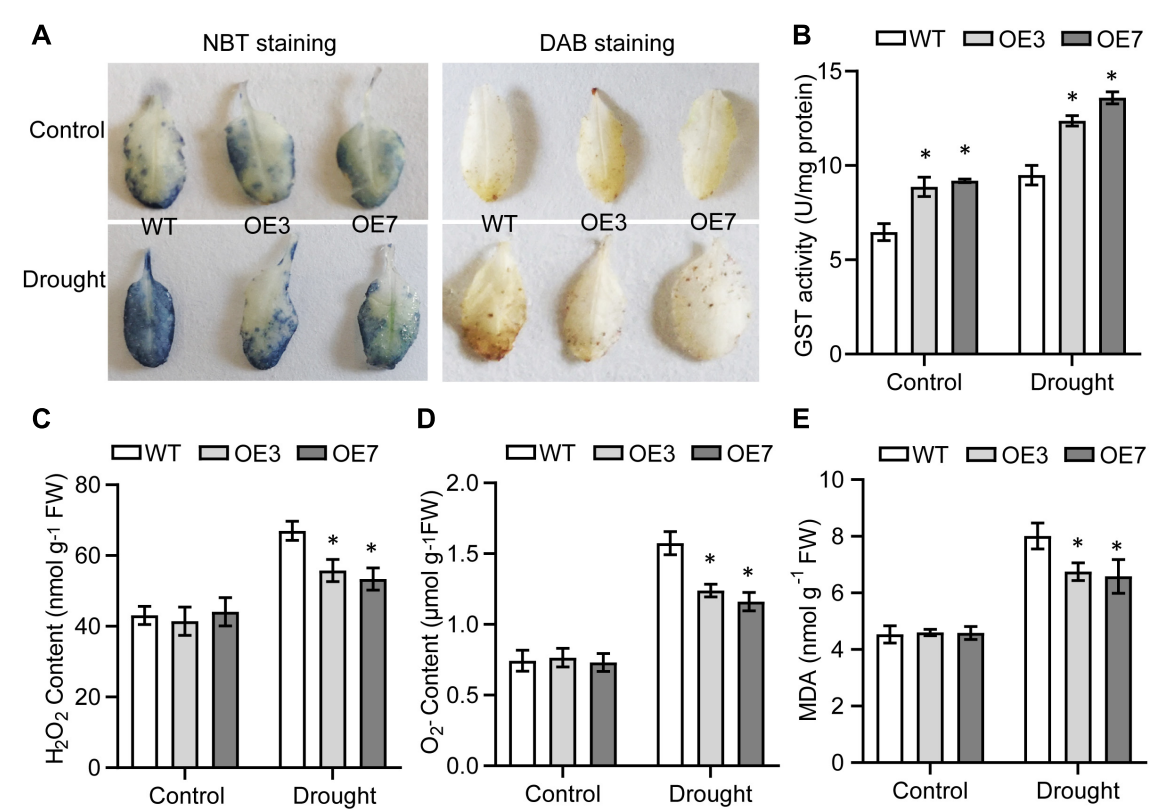

FIGURE 4 | Overexpression of CsGSTU8 alleviated ROS and MDA accumulation under drought stress. (A) Histochemical detection of $\mathrm{O}^{2-}$ and $\mathrm{H}_{2} \mathrm{O}_{2}$ via $\mathrm{NBT}^{-}$ staining and DAB staining, respectively. (B) Measurement of GST activity under control and drought conditions. (C) Measurement of $\mathrm{H}_{2} \mathrm{O}_{2}$ content under control and drought conditions. (D) Measurement of $\mathrm{O}^{2-}$ content under control and drought conditions. (E) Measurement of MDA content under control and drought conditions. The Data are presented as the means \pm SDs of three independent experiments. Significant differences were determined using Student's $t$-test $(* P<0.05)$.

CsGSTU8 might be regulated by WRKY transcription factors. Remarkably, our results revealed that the expression of CsGSTU8 similar to that of CsWRKY48, was induced in response to both drought and ABA treatment, and we inferred that CsWRKY48 may be involved in the regulation of CsGSTU8 expression. Thus, EMSAs and dual-LUC transient expression assays were carried out to verify this hypothesis. First, GST and GST-CsWRKY48 were purified for use in EMSAs and the band shifts were observed when the GST-CsWRKY48 protein was incubated together with a biotin-labeled probe (containing the core sequences (TGAC) of W-boxes), while band shifts were not observed in the GST protein control (Figure 6A), which indicated that CsWRKY48 can directly bind to the CsGSTU8 promoter. Dual-LUC transient expression assays showed that the transient expression of CsWRKY48 significantly increased the LUC/REN ratio of the reporter relative to that of the corresponding empty control in tobacco leaves (Figures 6B-D). In addition, we transiently overexpressed CsWRKY48 in tea plant leaves (Figures 6E,F), and the qRT-PCR results showed that CsWRKY48 overexpression increased the expression of CsGSTU8 in tea plant (Figure 6G). Taken together, our results indicate that CsWRKY48 can directly bind to the promoter of CsGSTU8 and activate its expression.

\section{DISCUSSION}

GSTs with a wide range of functions in plants, including protecting cells from oxidative damage during stresses (Sheehan et al., 2001). A series of studies have indicated that the expression of GST genes is induced in response to drought stress in various plants, including barley (Rezaei et al., 2013), tomato (Xu et al., 2015), wheat (Wang et al., 2019), potato (Islam et al., 2018), pepper (Islam et al., 2019), and rice (Jain et al., 2010). Previous studies in tea plant have reported that drought stress also induces the expression of members of CsGSTs (Parmar et al., 2019; Xia et al., 2019; Sun et al., 2020), including CsGSTU8, implying that GSTs from tea plant may have conserved functions in response to drought stress, similar to cases in other plants. Based on this information, we investigated the role and regulation of CsGSTU8 in response to drought stress in this study.

As it well established that abiotic stress, such as drought, salinity and cold alter the ABA levels in plants. ABA plays an important role as an essential mediator in triggering plant responses to various abiotic stresses (Hartung et al., 1988). Several GSTs have been reported to be associated with ABA signaling and to be involved in the stress response. For example, Sharma et al. (2014) reported that OsGSTU4 was induced in response to $\mathrm{ABA}$ and was involved in $\mathrm{ABA}$-dependent processes that provide stress tolerance to transgenic plants. In wheat, the TaERF3-actived TaGST6 expression was enhanced by ABA to respond to salt and drought stress (Rong et al., 2014). Our results indicated that the expression of CsGSTU8 was increased in response to $\mathrm{ABA}$ and drought treatments, meanwhile, the ABAand drought-responsive transcription factor CsWRKY48 could directly activate the expression of CsGSTU8. Thus, we inferred that ABA signaling might be involved in the drought-induced expression of CsGSTU8.

One of effects of drought stresses on plants is the excess accumulation of ROS. A series of studies have shown that transgenic plants expressing GSTs contribute to drought stress 


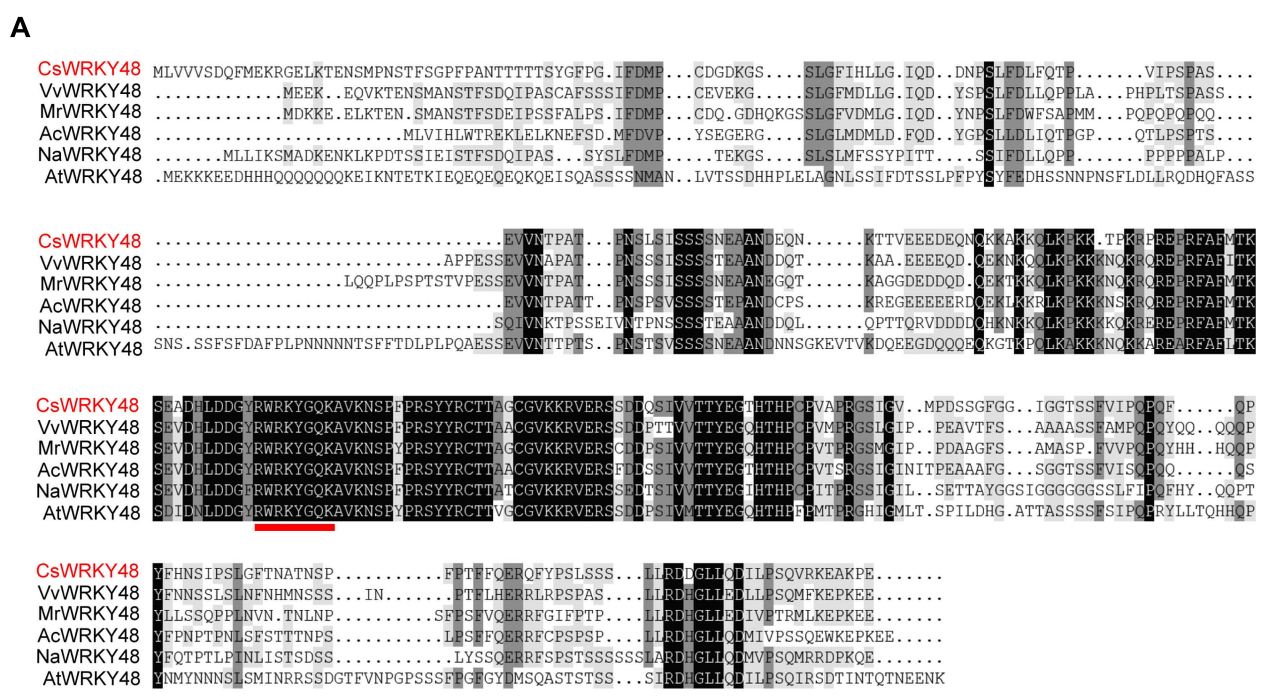

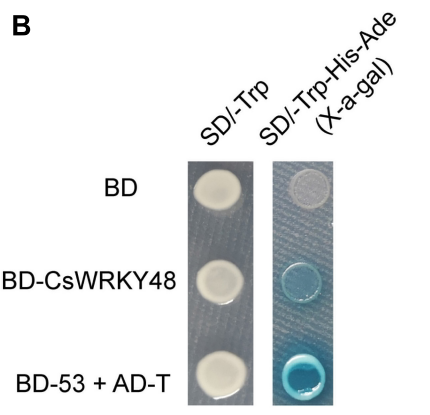

E

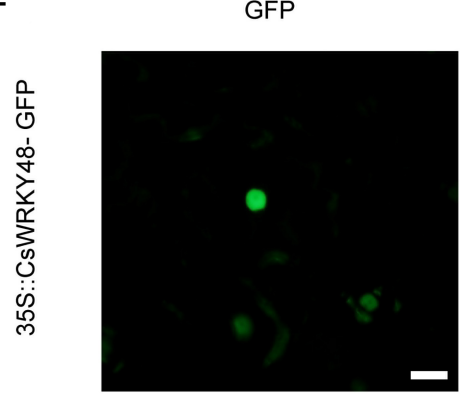

C

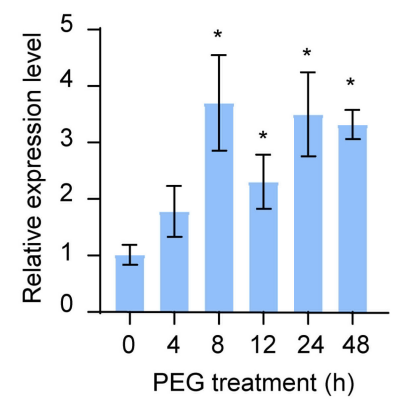

Bright

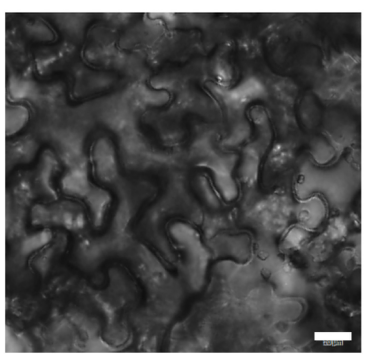

D
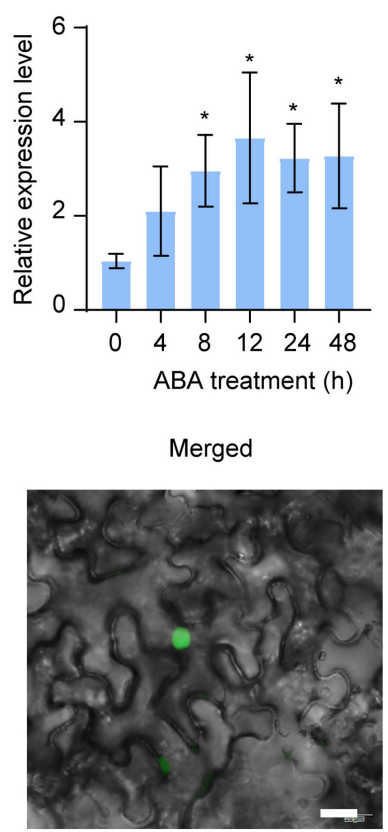

FIGURE 5 | CsWRKY48 acts as a transcriptional activator and is expressed in response to drought and ABA treatment. (A) Multiple sequence alignment of CsWRKY48 and its homologs in other plant species. The red line indicates the WRKY DNA-binding domain. (B) Transactivation analysis of CsWRKY48 in yeast. (C) Transcript levels of CsWRKY48 under PEG treatment. (D) Transcript levels of CsWRKY48 under ABA treatment. (E) Subcellular localization of 35S::CsWRKY48-GFP in tobacco cells. Bar $=20 \mu \mathrm{m}$. The data are presented as the means \pm SDs of three independent experiments. Significant differences were determined using Student's $t$-test $\left({ }^{\star} P<0.05\right)$.

tolerance by scavenging excess ROS accumulation (Liu et al., 2013a; Srivastava et al., 2019; Yang et al., 2019). Similarly, in the current study, transgenic Arabidopsis plants expressing CsGSTU8 exhibited a stress-tolerant phenotype when subjected to drought stress accompanied by less accumulation of ROS level, suggesting the positive role of CsGSTU8 in ROS scavenging. GST proteins catalyze the conjugation of GSH to an array of hydrophobic and electrophilic substrates, including ROS, thus, protecting the cell from oxidative burst (Kumar and Trivedi, 2018). During catalysis, the binding and correct orientation of GSH are governed by the conserved GSH binding site (G-site), and the substrate binding pocket (H-site) assists in the binding of substrates by providing a hydrophobic environment (Basantani and Srivastava, 2007; Kumar and Trivedi, 2018). CsGSTU8 harboring the conserved GSH bind site and the substrate binding pocket, suggesting the capacity of CsGSTU8 to catalyze the conjugation of GSH to an array of substrates, which was further confirmed by the higher GST activity of transgenic plants compared to that in WT plants. 


\section{A}

\section{B}

Probe:GTGGCTGCATGACATTATTITGCATCATGAAGTTGACGACCGACTG

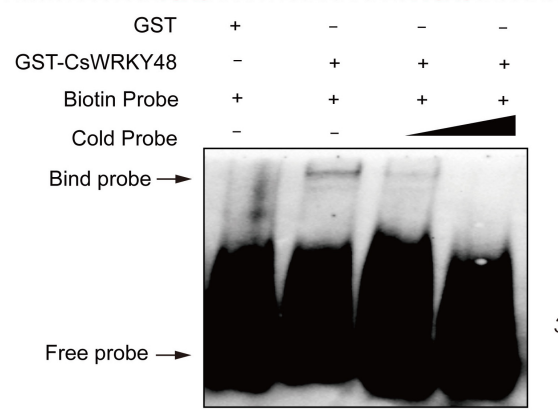

Reporter

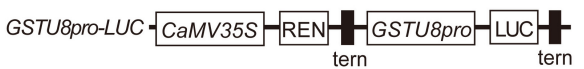

Effector

35 Empty - CaMV35S GFP 35 S tern-

35S-CSWRKY48 - CaMV35S -CsWRKY48 GFP 35 S tern-

C

D
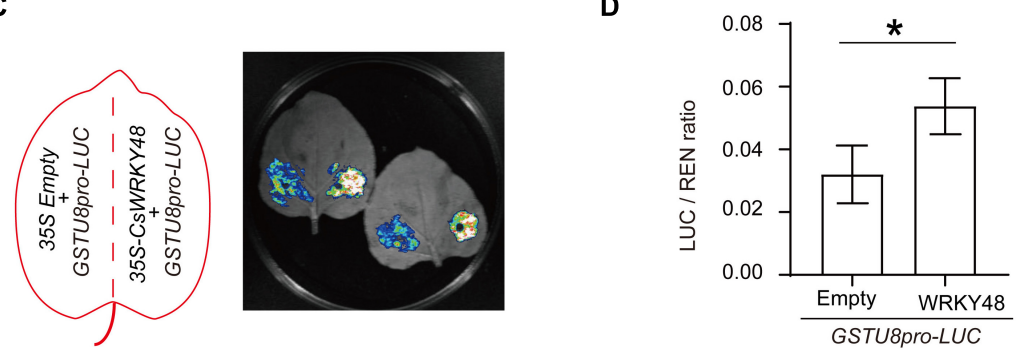

$\mathbf{E}$

$\mathbf{F}$

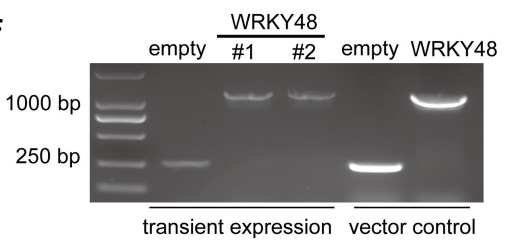

G

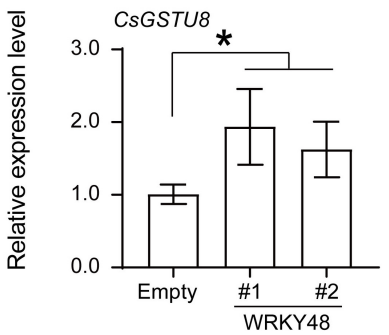

FIGURE 6 | CSWRKY48 binds to the promoter of CSGSTU8 and activates its expression. (A) EMSAs using purified GST and GST-CSWRKY48 proteins incubated together with biotin-labeled probes of W-box core elements (TGAC sequences) presented in CsGSTU8 promoter. -: absence; +: presence. (B) Schematics of the reporter and effector constructs used in the dual-LUC assays. (C) LUC signal detected in tobacco leaves. (D) LUC/REN ratio, as measured by the dual-LUC assays. (E) Transient expression of 35S::CsWRKY48-GFP and 35S::GFP in tea plant leaves. (F) RT-PCR was used to identify the transformation of 35S::CsWRKY48-GFP and 35S::GFP by using specific primers. (G) Expression of CsGSTU8 in transformed tea plant leaves. The data are presented as the means \pm SDs of three independent experiments. Significant differences were determined using Student's $t$-test $\left({ }^{*} P<0.05\right)$.

Thus, the lower ROS level of transgenic plants under drought stress can be explained by the fact that transgenic plants have an improved capacity for GSH conjugation to ROS and result in an enhanced ability to scavenge ROS, which constitutes the foundation for their stress-tolerant phenotype.

During signal transduction and the adaptive response, transcription factors including NAC, MYB, bZIP, DREB, and WRKY ones usually bind to the promoters of effector genes that encode enzymes, chaperones and ion/water channels and directly regulate their expression to induce an adaptive response under drought conditions (Joshi et al., 2016; Wei et al., 2019).
As effector genes encoding enzymes involved in ROS scavenging under stress, GSTs have been found to be directly regulated by several type transcription factors in response to drought stress in plants, such as ERF type (Rong et al., 2014), bHLH type (Cui et al., 2019), and NAC type (Ren et al., 2020). WRKY family members regulate the expression of target genes by binding to the W-box cis-elements in their promoters (Deng et al., 2020; Zhao et al., 2020). For example, AtWRKY57 can directly bind to the W-box in AtRD29A and AtNCED3 promoters and activate their expression (Jiang et al., 2012). Our EMSA and Dual-LUC transient expression assays demonstrated that a drought- and 
ABA-responsive transcription factor form tea plant, CsWRKY48, can directly bind to the W-box in promoter of CsGSTU8 and activate its expression. These results suggest that drought stress may first induces the expression of CsWRKY48 and then activates the CsGSTU8 transcription, thus causing an adaptive response.

\section{CONCLUSION}

In conclusion, our study revealed that CsGSTU8, which is positively activated by CsWRKY48, enhances the drought tolerance of transgenic Arabidopsis by increasing ROS scavenging under drought stress. This study provides valuable knowledge for understanding the function of CsGSTU8 and the underlying molecular mechanism of drought tolerance in tea plant.

\section{DATA AVAILABILITY STATEMENT}

The original contributions presented in the study are included in the article/Supplementary Material, further inquiries can be directed to the corresponding author/s.

\section{REFERENCES}

Basantani, M., and Srivastava, A. (2007). Plant glutathione transferases - a decade falls short. Canadian J. Botany 85, 443-456.

Carvalho, M. H. C. D. (2008). Drought stress and reactive oxygen species: production, scavenging and signaling. Plant Signal. Behav. 7, 599-601. doi: $10.4161 /$ psb.3.3.5536

Choudhury, F. K., Rivero, R. M., Blumwald, E., and Mittler, R. (2017). Reactive oxygen species, abiotic stress and stress combination. Plant J. 90, 856-867. doi: $10.1111 /$ tpj.13299

Cui, X. Y., Gao, Y., Guo, J., Yu, T. F., Zheng, W. J., Liu, Y. W., et al. (2019). BES/BZR transcription factor TaBZR2 positively regulates drought responses by activation of TaGST1. Plant Physiol. 180, 605-620. doi: 10.1104/pp.19.00100

Deng, B., Wang, W., Ruan, C., Deng, L., Yao, S., and Zeng, K. (2020). Involvement of CsWRKY70 in salicylic acid-induced citrus fruit resistance against Penicillium digitatum. Hortic. Res. 7:157.

Draper, H. H., Squires, E. J., Mahmood, H., Wu, J., Agarwa, S., and Hadley, M. (1992). A comparative evaluation of thiobarbituric acid methods for the determination of malondialdehyde in biological materials. Free Radic. Biol. Med. 15, 353-363. doi: 10.1016/0891-5849(93)90035-s

Gill, S. S., and Tuteja, N. (2010). Reactive oxygen species and antioxidant machinery in abiotic stress tolerance in crop plants. Plant Physiol. Biochem. 48, 909-930. doi: 10.1016/j.plaphy.2010.08.016

Hartung, W., Radin, J. W., and Hendrix, D. L. (1988). Abscisic acid movement into the apoplastic solution of water-stresses cotton leaves. Plant Physiol. 86, 908-913.

Islam, M. S., Choudhury, M., Majlish, A. K., Islam, T., and Ghosh, A. (2018). Comprehensive genome-wide analysis of Glutathione S-transferase gene family in potato (Solanum tuberosum L.) and their expression profiling in various anatomical tissues and perturbation conditions. Gene 639, 149-162. doi: 10 . 1016/j.gene.2017.10.007

Islam, S., Sajib, S. D., Jui, Z. S., Arabia, S., Islam, T., and Ghosh, A. (2019). Genome-wide identification of glutathione S-transferase gene family in pepper, its classification, and expression profiling under different anatomical and environmental conditions. Sci. Rep. 9:9101. doi: 10.1038/s41598-019-45320-x

Jain, M., Ghanashyam, C., and Bhattacharjee, A. (2010). Comprehensive expression analysis suggests overlapping and specific roles of rice glutathione S-transferase

\section{AUTHOR CONTRIBUTIONS}

YY and YoZ designed the experiments. YoZ and JH performed the experiments and data analysis. YoZ and YX wrote the manuscript. SW, YiZ, and YL provided valuable advice on the manuscript. LL, YD, and HL revised the manuscript. All authors contributed to the article and approved the submitted version.

\section{FUNDING}

This work was supported by the China Agriculture Research System of MOF and MARA (CARS-19), the Agricultural Special Fund Project of Shaanxi Province (NYKJ-2020-YL-13), the special fund for University-Supported Extension Model (XTG2021-04), and Key R\&D Program of Shaanxi Province (2020LSFP3-16).

\section{SUPPLEMENTARY MATERIAL}

The Supplementary Material for this article can be found online at: https://www.frontiersin.org/articles/10.3389/fpls.2021. 795919/full\#supplementary-material

genes during development and stress responses. BMC Genomics 11:73. doi: 10.1186/1471-2164-11-73

Jambunathan, N. (2010). Determination and detection of reactive oxygen species (ROS), lipid peroxidation, and electrolyte leakage in plants. Methods Mol. Biol. 639, 292-298. doi: 10.1007/978-1-60761-702-0_18

Jefferson, R. A., Kavanagh, T. A., and Bevan, M. W. (1987). GUS fusions: betaglucuronidase as a sensitive and versatile gene fusion marker in higher plants. EMBO J. 6, 3901-3907.

Jha, B., Sharma, A., and Mishra, A. (2011). Expression of SbGSTU (tau class glutathione S-transferase) gene isolated from Salicornia brachiata in tobacco for salt tolerance. Mol. Biol. Rep. 38, 4823-4832. doi: 10.1007/s11033-010-0625-x

Jiang, Q., Yang, J., Wang, Q., Zhou, K., Mao, K., and Ma, F. (2019). Overexpression of MdEPF2 improves water use efficiency and reduces oxidative stress in tomato. Environ. Exp. Bot. 162, 321-332. doi: 10.1007/s00394-014-0770-4

Jiang, Y., Liang, G., and Yu, D. (2012). Activated expression of WRKY57 confers drought tolerance in Arabidopsis. Mol. Plant 5, 1375-1388. doi: 10.1093/mp/ sss080

Joshi, R., Wani, S. H., Singh, B., Bohra, A., Dar, Z. A., Lone, A. A., et al. (2016). Transcription factors and plants response to drought stress: current understanding and future directions. Front. Plant Sci. 7:1029. doi: 10.3389/fpls. 2016.01029

Kumar, S., Asif, M. H., Chakrabarty, D., Tripathi, R. D., Dubey, R. S., and Trivedi, P. K. (2013). Expression of a rice Lambda class of glutathione S-transferase, OsGSTL2, in Arabidopsis provides tolerance to heavy metal and other abiotic stresses. J. Hazard. Mater. 248-249, 228-237. doi: 10.1016/j.jhazmat.2013.01. 004

Kumar, S., and Trivedi, P. K. (2018). Glutathione S-Transferases: role in combating abiotic stresses including arsenic detoxification in plants. Front. Plant Sci. 9:751. doi: 10.3389/fpls.2018.00751

Liu, D., Liu, Y., Rao, J., Wang, G., Li, H., Ge, F., et al. (2013a). Overexpression of the glutathione S-transferase gene from Pyrus pyrifolia fruit improves tolerance to abiotic stress in transgenic tobacco plants. Mol. Biol. 47, 515-523. doi: 10.7868/ s0026898413040101

Liu, Y. J., Han, X. M., Ren, L. L., Yang, H. L., and Zeng, Q. Y. (2013b). Functional divergence of the glutathione S-transferase supergene family in Physcomitrella patens reveals complex patterns of large gene family evolution in land plants. Plant Physiol. 161, 773-786. doi: 10.1104/pp.112.205815 
Moller, I. M., Jensen, P. E., and Hansson, A. (2007). Oxidative modifications to cellular components in plants. Annu. Rev. Plant Biol. 58, 459-481. doi: 10.1146/ annurev.arplant.58.032806.103946

Parmar, R., Seth, R., Singh, P., Singh, G., Kumar, S., and Sharma, R. K. (2019). Transcriptional profiling of contrasting genotypes revealed key candidates and nucleotide variations for drought dissection in Camellia sinensis (L.) O. Kuntze. Sci. Rep. 9:7487. doi: 10.1038/s41598-019-43925-w

Qi, J., Song, C. P., Wang, B., Zhou, J., Kangasjarvi, J., Zhu, J. K., et al. (2018). Reactive oxygen species signaling and stomatal movement in plant responses to drought stress and pathogen attack. J. Int. Plant Biol. 60, 805-826. doi: 10.1111/jipb.12654

Ren, Z., Zhang, D., Cao, L., Zhang, W., Zheng, H., Liu, Z., et al. (2020). Functions and regulatory framework of ZmNST3 in maize under lodging and drought stress. Plant Cell Environ. 43, 2272-2286. doi: 10.1111/pce.13829

Rezaei, M. K., Shobbar, Z. S., Shahbazi, M., Abedini, R., and Zare, S. (2013). Glutathione S-transferase (GST) family in barley: identification of members, enzyme activity, and gene expression pattern. J. Plant Physiol. 170, 1277-1284. doi: 10.1016/j.jplph.2013.04.005

Rong, W., Qi, L., Wang, A., Ye, X., Du, L., Liang, H., et al. (2014). The ERF transcription factor TaERF3 promotes tolerance to salt and drought stresses in wheat. Plant Biotechnol. J. 12, 468-479. doi: 10.1111/pbi.12153

Samarina, L. S., Bobrovskikh, A. V., Doroshkov, A. V., Malyukova, L. S., Matskiv, A. O., Rakhmangulov, R. S., et al. (2020). Comparative expression analysis of stress-inducible candidate genes in response to cold and drought in tea plant [Camellia sinensis (L.) Kuntze]. Front. Genet. 11:611283. doi: 10.3389/fgene. 2020.611283

Sasan, M., Maryam, E., Fateme, M., Maryam, S., Babak, S., and Hassan, M. (2011). Plant glutathione S-transferase classification, structure and evolution. African J. Biotechnol. 10, 8160-8165.

Shao, H. B., Chu, L. Y., Jaleel, C. A., and Zhao, C. X. (2008). Water-deficit stressinduced anatomical changes in higher plants. C. R. Biol. 331, 215-225. doi: 10.1016/j.crvi.2008.01.002

Sharma, R., Sahoo, A., Devendran, R., and Jain, M. (2014). Over-expression of a rice tau class glutathione s-transferase gene improves tolerance to salinity and oxidative stresses in Arabidopsis. PLoS One 9:e92900. doi: 10.1371/journal.pone. 0092900

Sheehan, D., Meade, G., Foley, V. M., and Dowd, C. A. (2001). Structure, function and evolution of glutathione transferases: implications for classification of nonmammalian members of an ancient enzyme superfamily. Biochem J. 360, 1-16. doi: 10.1042/bj3600001

Shi, J., Fu, X. Z., Peng, T., Huang, X. S., Fan, Q. J., and Liu, J. H. (2010). Spermine pretreatment confers dehydration tolerance of citrus in vitro plants via modulation of antioxidative capacity and stomatal response. Tree Physiol. 30, 914-922. doi: 10.1093/treephys/tpq030

Shui, L. Y., Yan, M. L., Li, H., Wang, P., Zhao, H., Wang, M. L., et al. (2021). Characterization of the R2R3-MYB Transcription Factor CsMYB113 Regulates Anthocyanin Biosynthesis in Tea Plants (Camellia Sinensis). PREPRINT (Version 1). Durham, NC: Research Square.

Singh, A., Kumar, A., Yadav, S., and Singh, I. K. (2019). Reactive oxygen speciesmediated signaling during abiotic stress. Plant Gene 18, 405-413.

Smirnoff, N. (1993). The role of active oxygen in the response of plants to water deficit and desiccation. New Phytol. 125, 27-58. doi: 10.1111/j.1469-8137.1993. tb03863.x

Srivastava, D., Verma, G., Chauhan, A. S., Pande, V., and Chakrabarty, D. (2019). Rice (Oryza sativa L.) tau class glutathione S-transferase (OsGSTU30) overexpression in Arabidopsis thaliana modulates a regulatory network leading to heavy metal and drought stress tolerance. Metallomics 11, 375-389. doi: 10.1039/c8mt00204e

Stavridou, E., Voulgari, G., Michailidis, M., Kostas, S., Chronopoulou, E. G., Labrou, N. E., et al. (2021). Overexpression of a biotic stress-inducible pvgstu gene activates early protective responses in tobacco under combined heat and drought. Int. J. Mol. Sci. 22:2352. doi: 10.3390/ijms22052352

Sun, J., Qiu, C., Ding, Y., Wang, Y., Sun, L., Fan, K., et al. (2020). Fulvic acid ameliorates drought stress-induced damage in tea plants by regulating the ascorbate metabolism and flavonoids biosynthesis. BMC Genomics 21:411. doi: 10.1186/s12864-020-06815-4

Wan, S., Zhang, Y., Duan, M., Huang, L., Wang, W., Xu, Q., et al. (2020). Integrated analysis of long non-coding RNAs (lncRNAs) and mRNAs reveals the regulatory role of lncRNAs associated with salt resistance in Camellia sinensis. Front. Plant Sci. 11:218. doi: 10.3389/fpls.2020.00218

Wang, R., Ma, J., Zhang, Q., Wu, C., Zhao, H., Wu, Y., et al. (2019). Genome-wide identification and expression profiling of glutathione transferase gene family under multiple stresses and hormone treatments in wheat (Triticum aestivum L.). BMC Genomics 20:986. doi: 10.1186/s12864-019-6374- $\mathrm{x}$

Wei, W., Liang, D. W., Bian, X. H., Shen, M., Xiao, J. H., Zhang, W. K., et al. (2019). GmWRKY54 improves drought tolerance through activating genes in abscisic acid and Ca2+ signaling pathways in transgenic soybean. Plant J. 100, 384-398. doi: 10.1111/tpj.14449

Xia, E. H., Li, F. D., Tong, W., Li, P. H., Wu, Q., Zhao, H. J., et al. (2019). Tea plant information archive: a comprehensive genomics and bioinformatics platform for tea plant. Plant Biotechnol. J. 17, 1938-1953. doi: 10.1111/pbi.13111

Xiao, L., Tang, L., Wang, W., Gao, Y., Huang, Y., Meng, Y., et al. (2020). Cloning and functional analysis of CsWRKYIIcs transcription factors in tea plant. Sci. Agricult. Sinica 53, 2460-2476. doi: 10.1016/j.gene.2017.05.044

Xu, J., Tian, Y. S., Xing, X. J., Peng, R. H., Zhu, B., Gao, J. J., et al. (2016). Over-expression of AtGSTU19 provides tolerance to salt, drought and methyl viologen stresses in Arabidopsis. Physiol. Plant. 156, 164-175. doi: 10.1111/ppl. 12347

Xu, J., Xing, X. J., Tian, Y. S., Peng, R. H., Xue, Y., Zhao, W., et al. (2015). Transgenic Arabidopsis plants expressing tomato glutathione S-Transferase showed enhanced resistance to salt and drought stress. PLoS One 10:e136960. doi: 10.1371/journal.pone.0136960

Yang, G., Wang, Y., Xia, D., Gao, C., Wang, C., and Yang, C. (2014). Overexpression of a GST gene (ThGSTZ1) from Tamarix hispida improves drought and salinity tolerance by enhancing the ability to scavenge reactive oxygen species. Plant Cell Tissue Organ. Culture 117, 99-112.

Yang, Q., Liu, Y.-J., and Zeng, Q. Y. (2019). Overexpression of three orthologous glutathione S-transferases from populus increased salt and drought resistance in Arabidopsis. Biochem. Systemat. Ecol. 83, 57-61.

Zhang, X., Henriques, R., Lin, S. S., Niu, Q. W., and Chua, N. H. (2006). Agrobacterium-mediated transformation of Arabidopsis thaliana using the floral dip method. Nat. Protoc. 1, 641-646. doi: 10.1038/nprot.2006.97

Zhang, Y., Wan, S., Liu, X., He, J., Cheng, L., Duan, M., et al. (2020). Overexpression of CsSnRK2.5 increases tolerance to drought stress in transgenic Arabidopsis. Plant Physiol. Biochem. 150, 162-170. doi: 10.1016/j. plaphy.2020.02.035

Zhao, X., Qi, C., Jiang, H., Zhong, M., You, C., Li, Y., et al. (2020). MdWRKY15 improves resistance of apple to Botryosphaeria dothidea via the salicylic acidmediated pathway by directly binding the MdICS1 promoter. J. Integr. Plant Biol. 62, 527-543. doi: 10.1111/jipb.12825

Conflict of Interest: The authors declare that the research was conducted in the absence of any commercial or financial relationships that could be construed as a potential conflict of interest.

Publisher's Note: All claims expressed in this article are solely those of the authors and do not necessarily represent those of their affiliated organizations, or those of the publisher, the editors and the reviewers. Any product that may be evaluated in this article, or claim that may be made by its manufacturer, is not guaranteed or endorsed by the publisher.

Copyright (c) 2021 Zhang, He, Xiao, Zhang, Liu, Wan, Liu, Dong, Liu and Yu. This is an open-access article distributed under the terms of the Creative Commons Attribution License (CC BY). The use, distribution or reproduction in other forums is permitted, provided the original author(s) and the copyright owner(s) are credited and that the original publication in this journal is cited, in accordance with accepted academic practice. No use, distribution or reproduction is permitted which does not comply with these terms. 